\title{
Microscopic marvels
}

\author{
Microscopes are changing the face of biology. Researchers should innovate and collaborate \\ if they want to be part of the new vision.
}

W atching molecular-scale events unfold in a living cell can be an inspiring experience. The inner workings of the nucleus, the shuttling of cellular cargo, the passage of messages through a membrane - seeing this tumultuous activity up close can fire the scientific imagination in a way that abstract data from genetic sequences or chemical analyses can never quite equal.

This helps to explain why microscopes are such essential tools in science, and why scientists' desire to see more is driving rapid innovation in the field. Five microscopes representative of these innovations are featured in this issue, starting on page 629. They range from 'super-resolution' devices that use light to reveal details once visible only with electron microscopes, to an electron microscope that can peer into thick samples once accessible only to optical ones.

Few of these new imaging technologies come cheaply, however. One new-generation light microscope can easily cost US\$500,000 or more, not counting the staff and training required to use it. There is no room for error on an instrument so sensitive that the slightest vibration or misstep in the experimental set-up creates artefacts.

As a result, biologists may have to get used to sharing their instruments. Research groups often prefer to buy their own microscopes so that lab members can use them whenever they want. But to stay at the forefront of image-led science, they will increasingly have to work with their departments, universities and funding agencies to create shared microscopy facilities staffed by specialists.

Getting engineers into the mix will be a good idea, and computational biologists will be a must. Automation, computer processing and image reconstruction are now central to microscopy and imaging. This means that there is increasing separation between the sample placed in the microscope and what scientists actually see, and that there are numerous points at which inaccuracies can inadvertently be introduced. The only way to interpret the images the system spits out is to understand (and explain alongside the final results) precisely what went into their creation. Not that this problem is new: scientists

\section{Stem-cell clarity}

\section{The draft NIH guidelines on stem-cell research are a good first step, but some revision is needed.}

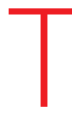
he proposed guidelines on federal funding for stem-cell research issued in April by the US National Institutes of Health $(\mathrm{NIH})$ are a welcome effort to assert ethical and regulatory leadership over this field - especially given the vacuum in oversight left by the previous US administration. Yet concerns aired by the scientific community during the public comment period that closed last week have underscored the need for the NIH to revise the have struggled with how to interpret microscope images since the instrument's earliest days (see page 642).

As well as sharing microscopes, cell and molecular biologists will need to share the instruments' output. A good way to do that would be through a central repository or framework for biological images modelled on existing data repositories such as GenBank. Such a resource would not only help to make the images universally available — using widely accepted data standards — but could be a driver for the development of analysis techniques that extract deeper meaning from existing images. A database launched last year by The Journal of Cell Biology (http://jcb-dataviewer. rupress.org) is already a start towards this goal. Such experiments deserve the community's support and participation.

Meanwhile, some labs are exploring an
"The era of systems microscopy could soon be here." alternative to the fat-price-tag rule: instead of buying one machine for $\$ 500,000$, buy 50,000 microscopes for $\$ 10$ apiece. By creating microscopes that are small, cheap and even disposable, these researchers hope to accelerate the development of microscopy into a highthroughput, automated procedure that can quickly collect data on living cells as systematic changes are made to one gene, protein or receptor at time. The era of systems microscopy could soon be here.

The wonderful thing about thinking visually is that it is so easy to think big. And that's exactly what researchers should do as they move ahead. By thinking about what they really want to see, they will help to devise microscopes - as well as computation, labelling and samplepreparation techniques - that make that vision possible. Thinking big might lead to microscopy that allows individual molecules to be tracked across thousands of cells in real time in living tissue; that can watch a single cell over the months or years that lapse from birth to death; or that can map the intricate form of every neuron in the brain across multiple species. The deeper biologists look, the more they will find there is to see.

guidelines to allow the responsible progress of research.

For example, the NIH has acted admirably in setting forth nine strict informed-consent provisions regarding the source material for stem-cell lines that are eligible for federal funding. As currently written, however, the provisions would probably exclude funding for most embryonic stem cells now in use, because the cells were derived from leftover embryos at fertility clinics under rules less stringent than the ones now called for. In particular, one provision requires that embryo donors affirm that they are donating "without any restriction or direction" regarding the patients who may benefit from the research. Few of the consent forms currently in use by fertility clinics ask for that affirmation. Also absent from many forms is the stipulation that the donors "would not receive financial or any other 